\title{
Effect of Guizhifulingwan (Keishibukuryogan) on climacteric syndrome: study protocol for a randomized controlled pilot trial
}

\author{
Jung-Eun Kim¹, Junghyo Cho², Ojin Kwon', Ae-Ran Kim, Hyo-Ju Park', So-Young Jung', Joo-Hee Kim¹,
} Mikyung Kim', Hye-Yoon Lee ${ }^{1}$ and Jun-Hwan Lee ${ }^{1,3^{*}}$ (ID

\begin{abstract}
Background: The aim of this study is to explore the efficacy of Guizhifulingwan (GFW) in the treatment of climacteric syndrome in women.

Methods/design: This is a single-center, randomized, placebo-controlled, parallel-group design pilot trial. Fifty participants with climacteric syndrome will be randomly allocated to the GFW or placebo group in a 1:1 ratio. The participants will be administered GFW or placebo granules three times a day for 4 weeks and will be followed up for a further 4 weeks. The primary outcome is the mean change in menopause rating scale score at 5 weeks after randomization. Secondary outcomes include the World Health Organization quality of life-BREF scores, degrees of upward movement of qi and lower abdominal resistance and tenderness, blood stasis pattern questionnaire scores, and results of blood tests including assays for lipid profile, high sensitivity C-reactive protein, follicle-stimulating hormone, and estradiol. The feasibility outcomes include recruitment and completion rates and adherence to medication.
\end{abstract}

Discussion: The results of this study will provide basic data for the design of a large-scale clinical trial for evaluating the efficacy of GFW in the treatment of climacteric syndrome in women.

Trial registration: Clinical Research Information Service (CRIS), Republic of Korea, KCT0002040. Registered on 5 September 2016.

Keywords: Guizhifulingwan, Keishibukuryogan, Climacteric syndrome, Menopause, Randomized controlled trial

\section{Background}

Although menopause is a natural phenomenon in women, the accompanying pre- and post-menopausal symptoms can considerably affect their daily activities and quality of life (QOL) [1]. Although hormone replacement therapy (HRT) is known to be effective for menopausal symptoms [2], it has been reported to increase the incidence of thrombosis, stroke, and ovarian cancer [3, 4].

Because of concerns regarding the potential adverse events (AEs) associated with HRT, interest in and the

\footnotetext{
* Correspondence: omdjun@kiom.re.kr

${ }^{1}$ Clinical Research Division, Korea Institute of Oriental Medicine, 1672

Yuseongdae-ro, Yuseong-gu, Daejeon 34054, Republic of Korea

${ }^{3}$ University of Science \& Technology (UST), Korean Medicine Life Science,

Daejeon 34054, Republic of Korea

Full list of author information is available at the end of the article
}

use of complementary and alternative medicines related to climacteric symptoms are increasing. Although herbal medicine is among the more commonly used complementary and alternative medicines, there is little evidence supporting its efficacy $[5,6]$. Guizhifulingwan (GFW; Keishibukuryogan in Japanese) has been widely used for centuries in China, Korea, and Japan for gynecological disorders caused by blood stasis [7-10]. It also has been considered effective in the management of climacteric symptoms. The symptoms for which GFW is indicated-blood stasis pattern (hot flushes with cold legs, neck ache, and stiffness), solid constitution (not in a physically weakened state), ruddy face, etc.- are not necessarily restricted to women in menopausal transition; they are generally regarded as characteristic of 
women suffering from vasomotor symptoms such as hot flushes and perspiration $[11,12]$.

Although recent clinical studies have evaluated the efficacy of GFW in the treatment of menopause-related symptoms [13-15], a randomized controlled trial (RCT) for validating the same has not yet been undertaken. Although one of the relevant studies demonstrated a positive outcome in terms of QOL in menopausal women upon treatment with GFW, the study neither included a control group nor described the criteria for blood stasis pattern during participant selection [13]. On the other hand, while a previous RCT on hot flushes in menopausal women did not yield a positive outcome in this regard, it described the importance of symptom patterns of each participant in the inclusion criteria [16]. We designed this study to better reflect the characteristics of traditional medicine by considering the traditional symptom pattern of GFW in the participant inclusion criteria and assessment methods.

\section{Methods/design \\ Objective}

The aim of this study is to explore the efficacy of GFW in the treatment of climacteric syndrome in women.

\section{Design}

This study will be a randomized, double-blind, controlled, parallel-group pilot trial. Participants will be randomly assigned to the GFW or placebo group in a ratio of 1:1. The study period will be about 8 weeks-4 weeks each of medication and follow-up. The design is summarized in Figs. 1 and 2. The study protocol (version 1.1, 25 July 2016) follows the Standard Protocol Items: Recommendations for Interventional Trials (SPIRIT) guidelines (see Additional file 1).

\section{Recruitment}

Participants will be recruited through advertisements in local newspapers, the subway, and our hospital.

\section{Participants Inclusion criteria}

Inclusion criteria for the study will be as follows: (1) age, 45-60 years; (2) menopausal women (including those with natural or induced menopause) with pre- and postmenopausal climacteric symptoms; (3) menopause rating scale (MRS) $[17,18]$ score $\geq 9$; (4) moderate to high physical strength; (5) moderate to high degree of upward movement of qi; (6) moderate to high degree of lower abdominal resistance and tenderness; and (7) willingness to provide written informed consent.

Natural menopause is considered to have occurred after 12 consecutive months of amenorrhea with no other obvious pathological or physiological cause. The term "climacteric" encompasses perimenopause by extending the duration of symptoms for a variable period long before and after menopause. The term "perimenopause" includes the period immediately prior to menopause and the first year after menopause [19].

GFW is generally used for those who are not in a physically weakened state [10]. The degree of physical strength of inclusion criterion 4 is evaluated by referencing body physique, nutritional status, and the results of exclusion criteria 6 and 7 together, and is scored on a 3 -point scale $(1=$ low, 2 = moderate, and $3=$ high $)$.

\section{Exclusion criteria}

Exclusion criteria for the study are as follows: (1) serious and unstable medical conditions; (2) severe mental disease; (3) aspartate aminotransferase (AST), alanine aminotransferase (ALT), alkaline phosphatase (ALP),

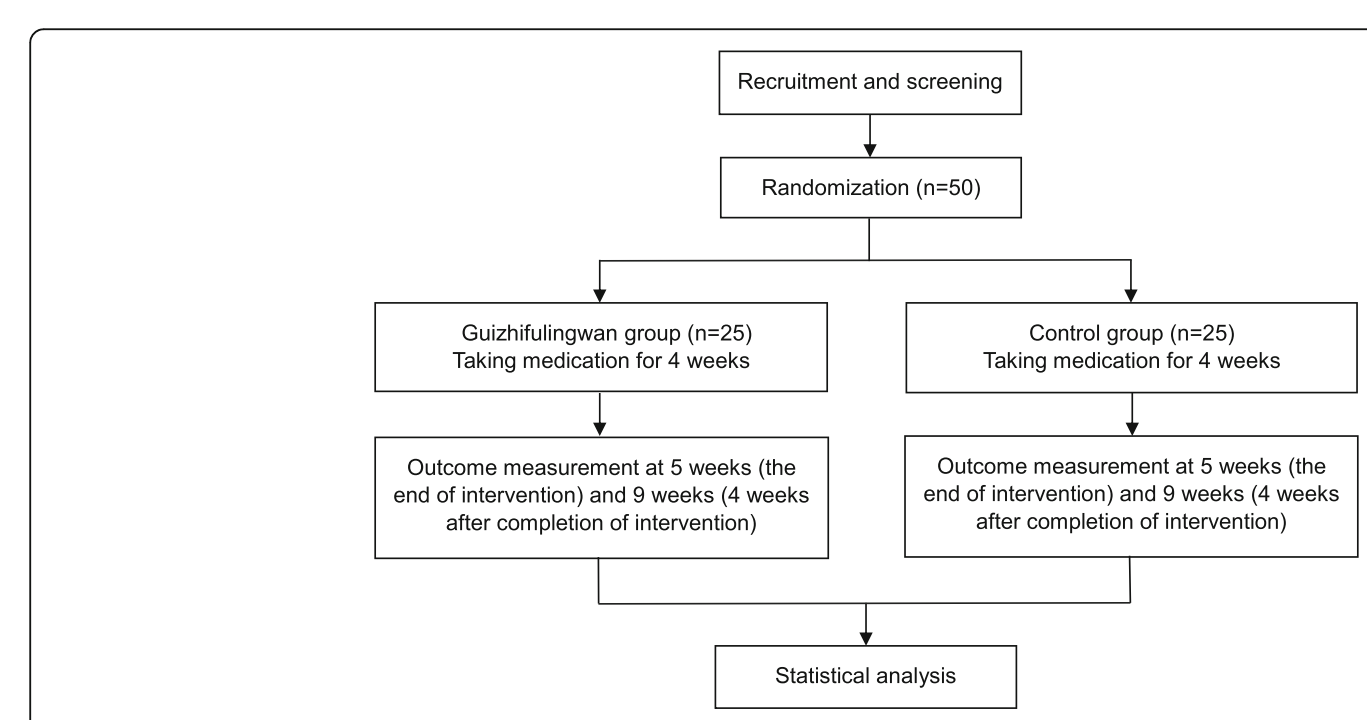

Fig. 1 Study flow chart 


\begin{tabular}{|c|c|c|c|c|c|c|}
\hline \multirow[b]{3}{*}{ TIMEPOINT** } & \multicolumn{6}{|c|}{ STUDY PERIOD } \\
\hline & \multirow{2}{*}{$\frac{\text { Enrolment }}{-t_{1}}$} & \multirow{2}{*}{$\begin{array}{l}\text { Allocation } \\
0 \text { (Week 1) }\end{array}$} & \multicolumn{3}{|c|}{ Post-allocation } & \multirow{2}{*}{$\begin{array}{c}\text { Close-out } \\
\text { Week } 9\end{array}$} \\
\hline & & & Week 1 & Week 3 & Week 5 & \\
\hline \multicolumn{7}{|l|}{ ENROLMENT: } \\
\hline $\begin{array}{r}\text { Eligibility screen } \\
\text { (Inclusion/exclusion } \\
\text { criteria) }\end{array}$ & $x$ & & & & & \\
\hline \multirow{2}{*}{$\begin{array}{r}\text { Informed consent } \\
\text { Random allocation }\end{array}$} & $x$ & & & & & \\
\hline & & $x$ & & & & \\
\hline \multicolumn{7}{|l|}{ INTERVENTIONS: } \\
\hline \multicolumn{7}{|l|}{$\begin{array}{r}\begin{array}{r}\text { Administration of } \\
\text { investigational } \\
\text { product }\end{array} \\
\end{array}$} \\
\hline \multicolumn{7}{|l|}{ ASSESSMENTS: } \\
\hline \multirow{2}{*}{$\begin{array}{r}\text { Demographic } \\
\text { characteristics } \\
\text { Medical history }\end{array}$} & $x$ & & & & & \\
\hline & $x$ & & & & & \\
\hline \multirow{3}{*}{$\begin{array}{r}\text { Physical examination } \\
\text { Laboratory test } \\
\text { Vital signs }\end{array}$} & $x$ & & & & & $x$ \\
\hline & $\mathrm{x}$ & & & & & $x$ \\
\hline & $\mathrm{x}$ & & $x$ & $x$ & $x$ & $x$ \\
\hline \multirow{3}{*}{$\begin{array}{r}\text { Concomitant therapy } \\
\text { Adverse events } \\
\text { Menopause rating } \\
\text { scale }\end{array}$} & & & $x$ & $x$ & $x$ & $x$ \\
\hline & & & $x$ & $x$ & $x$ & $x$ \\
\hline & $x$ & & & & $x$ & $x$ \\
\hline $\begin{array}{r}\text { Upward movement of } \\
q i\end{array}$ & $x$ & & & & $x$ & $x$ \\
\hline \multirow{2}{*}{$\begin{array}{r}\text { Lower abdominal } \\
\text { resistance and } \\
\text { tenderness } \\
\text { World Health } \\
\text { Oraanization auality }\end{array}$} & $x$ & & & & $x$ & $x$ \\
\hline & & & $x$ & & $x$ & $x$ \\
\hline \multirow{2}{*}{$\begin{array}{r}\text { Organization quality } \\
\text { of life-BREF } \\
\text { Questionnaire for } \\
\text { blood stasis pattern } \\
\text { Examination of } \\
\text { returned } \\
\text { investigational } \\
\text { product }\end{array}$} & & & $x$ & & $x$ & $x$ \\
\hline & & & & $x$ & $x$ & \\
\hline \multirow{2}{*}{$\begin{array}{r}\text { Medication adherence } \\
\text { Blinding test } \\
\end{array}$} & & & & $x$ & $x$ & \\
\hline & & & & & $x$ & \\
\hline \multicolumn{7}{|l|}{ OTHERS: } \\
\hline $\begin{array}{l}\text { Guidance for } \\
\text { visitation }\end{array}$ & $x$ & & $x$ & $x$ & $x$ & \\
\hline
\end{tabular}

Fig. 2 SPIRIT schedule for enrollment, interventions, and assessments

and $\gamma$-glutamyl transpeptidase $(\gamma$-GTP) levels 1.5 times as high as or higher than the normal upper limit; (4) blood urea nitrogen $(\mathrm{BUN})$ and creatinine $(\mathrm{Cr})$ levels 1.5 times as high as or higher than the normal upper limit; (5) thyroid-stimulating hormone level 1.5 times lower than the normal lower limit; (6) body mass index $<18.5$; (7) hemoglobin $(\mathrm{Hb})$ level $<11 \mathrm{~g} / \mathrm{dL}$; (8) estrogen therapy within a year prior to the study; (9) herbal medicine therapy related to climacteric syndrome within 4 weeks prior to the study; (10) vaginal bleeding without cause after menopause; (11) lactose intolerance; (12) participation in other clinical trials; (13) residents of collective dwelling facilities such as social welfare institutions; (14) unwillingness to provide written consent; and (15) participants judged unsuitable for the clinical trial by the investigator.

\section{Randomization and allocation concealment}

A statistician will generate random allocation numbers using a computer program (SAS [Strategic Applications Software], version 9.4; SAS Institute Inc., Cary, NC, USA). The generated numbers will be sealed in opaque envelopes and stored in double-locked cabinets. Participants who fulfill all of the inclusion criteria will be assigned to one of two groups by blocked randomization.

\section{Blinding}

The participants, investigators, coordinators, pharmacist, monitoring agent, and statistician will be blinded to the group allocation data, which will be known only to the person in charge of random allocation. The statistician will create a random allocation table indicating assignment to group $\mathrm{A}$ or $\mathrm{B}$. The person in charge of random 
allocation will deliver this allocation table and group information to the pharmaceutical company (as there is no one at the investigational products manufacturing company to create a randomization list, our team will prepare the randomization table). The pharmaceutical company will make and label investigational products based on this information. The label will include each participant's identification number (R1001 to R1050; identical to the random allocation numbers) and visit number (V1 or V2). An opaque emergency envelope containing allocation information will be prepared and stored in a safe place in anticipation of unexpected events [20]. Violation of blinding will be considered only under circumstances where knowledge of the medication being administered to a participant is essential for treatment. The validity of blinding will be assessed according to the new blinding index [21].

\section{Intervention}

Participants will be randomly assigned to the GFW or placebo control group in a ratio of 1:1. They will receive treatment or evaluation according to the predetermined schedule. All participants will orally ingest $3 \mathrm{~g}$ of granules three times a day for 4 weeks and present themselves for follow-up evaluation 4 weeks after the termination of medication. The drugs for this clinical trial will be provided to the participants twice in 2week intervals at weeks 1 and 3 . To confirm their adherence to the medication regimen, participants will be requested to return unused drugs as well as spent wrappers of used drugs.

Both GFW and the placebo are manufactured by Hanpoong Pharm. Co., Ltd. (Wanju, Republic of Korea) according to good manufacturing practice standards. Only those products that pass quality control tests will be used in this study. While GFW comprises Ramulus cinnamomi, Poria, Moutan cortex, Persicae semen, and Radix paeoniae [9] (3 g of GFW granules are prepared according to the granulation method using $1.33 \mathrm{~g}$ of GFW soft extract, which is prepared by extracting $1.33 \mathrm{~g}$ of each of the five herbs [same ratio] with water in an extractor, followed by filtration and concentration), the placebo comprises corn starch, lactose hydrate, citric acid hydrate, ginseng flavor powder, and caramel coloring. Both these drugs are similar in appearance and color (brown) and will be packaged in an identical shape.

\section{Concomitant treatment}

Participants of both groups will be prohibited from concomitant use of menopause-related hormone therapy (estrogen, estrogen/progestogen, or tibolone), nonprescription medicines, functional health foods, or Oriental medicines that can potentially affect the outcome of this experiment until after the follow-up period of 8 weeks.
Participants who have been on medication for chronic diseases prior to initiation of this study will be allowed to continue their regimen, and care will be taken not to alter the dosage or type of drug. Similarly, participants will be allowed to maintain their pre-trial exercise routines without altering the intensity or type of exercise. If the dosage or type of existing medication or intensity or type of exercise is changed, the participant will be required to report the same to the person in charge of this study.

\section{Outcome measurements}

The time points for the outcome measurements are presented in detail in Fig. 2.

\section{Primary outcome}

The primary outcome is the mean change in MRS score at week 5 . The MRS comprises 11 items encompassing 3 subscales: psychological, somatovegetative, and urogenital domains. Each item is scored on a 4-point scale, with scores ranging from 0 (no symptoms) to 4 (very severe). Based on the total score, determined by the sum of the scores of the subscales, the degree of symptoms can be classified as follows: "no, little" (0-4), "mild" (5-8), "moderate" (9-16), and "severe" (>17) [17, 18]. The Korean version of the MRS will be used in the present study (http://www.menopause-rating-scale.info).

\section{Secondary outcomes}

World Health Organization QOL-BREF (WHOQOLBREF) The WHOQOL-BREF comprises 26 questions including 24 questions regarding 4 sub-sections-physical and psychological health, social relationship, and environment-and 2 questions regarding overall QOL. Each item is scored on a 5-point scale as follows: 1 , strongly disagree; 2 , disagree; 3 , neutral; 4 , agree; and 5 , strongly agree. The Korean version of the WHOQOL-BREF will be used in the present study [22-24].

Degree of upward movement of qi The degree of upward movement of qi is evaluated by scoring symptoms corresponding to each of five body parts on a 5 -point scale (1, no symptoms; 2 , mild; 3 , moderate; 4 , severe; and 5, extreme). The sum of these scores is divided by 5 to obtain the final value for analysis. If the final value is a decimal, it is rounded off to one decimal place. The five body parts and their corresponding symptoms are as follows: head (headache, dizziness, and heavy-headedness); face (ruddy face and hot flushes); shoulders (neck and shoulder stiffness); chest (flushing sensation and palpitation); and lower limbs (cold legs and feet). These symptoms have been identified based on previous reports $[11,25,26]$, and 
the scoring method has been devised by the research team involved in this study.

Degree of lower abdominal resistance and tenderness Abdominal examination will be performed according to the following protocol. The practitioner's hands should be sufficiently warm before beginning the examination. The room itself should be warm enough that the participant does not shiver or become tense. The participant lies supine, comfortably, with both legs extended. When necessary, the exam can be conducted with both knees bent. The practitioner stands on the right (or left) side of the participant and first observes the entire abdomen and then palpates the lower abdomen while trying not to apply more pressure than necessary [25, 27]. The abdominal exam is performed from top to bottom and left to right. The abdominal symptoms of GFW include a resistance that feels like a palpable mass-like object in the lower abdomen. Tenderness is not severe [25, 26]. The degree of lower abdominal resistance and tenderness is scored on a 5-point scale (1, none; 2, mild; 3, moderate; 4 , severe; and 5, extreme).

Questionnaire for blood stasis pattern The questionnaire for blood stasis pattern comprises 14 items: sprain; contusion; old BiJeung (BiJeung shows symptoms similar to those of arthralgia and paresthesia syndrome [28]); stabbing pain; lower abdominal pain; hypochondriac pain; night pain; feeling of abdominal mass; easy congealing of blood; darkish complexion (discoloration of the face); purple and dull pallor of the mouth, lips, and tongue; bluish purple discoloration of the lower eyelid; darkish stools; and number of surgeries. Each item is scored on a 7-point scale [29].

Blood tests Blood tests including those for internal lipid (low-density lipoprotein, high-density lipoprotein, total cholesterol, and triglycerides), high sensitivity C-reactive protein (for prediction of risk of cardiovascular diseases [30]), and hormone (follicle-stimulating hormone and estradiol, for understanding the climacteric condition) levels will be conducted.

\section{Feasibility outcomes}

Recruitment rate The recruitment rate will be calculated as the percentage of participants selected from among those who undergo screening and the percentage of participants selected against the total number of participants.

Completion rate The completion rate will be calculated as the percentage of participants who complete the study without interruption until the endpoint against the total number of participants.
Medication adherence Medication adherence will be calculated as the percentage of doses ingested by the participants against the total dose.

\section{Safety and adverse event outcomes}

Adverse events (AEs) indicate undesirable and unintentional signs, symptoms, or diseases that develop after intervention during the period of a clinical trial. They do not necessarily have a causal relationship with the relevant intervention. Investigators will check participants' vital signs and examine for manifestation of AEs, including digestive symptoms, skin conditions, and jaundice, at every visit. Investigators will also conduct physical examination and laboratory tests, including evaluation of AST, ALT, ALP, $\gamma$-GTP, BUN, Cr, Hb, and hematocrit levels and red and white blood cell, differential, and platelet counts, at the screening as well as at the fourth visit (week 9). In case of AEs, the correlation between AEs and intervention will be classified into one of the following six categories: (1) definitely related, (2) appears to be related, (3) possibly related, (4) appears to be unrelated, (5) definitely not related, or (6) unclear.

\section{Sample size}

The null hypothesis $\left(H_{O}\right)$ of this study is that there will be no difference in the mean change in MRS scores between the GFW and placebo groups after 4 weeks of medication. The alternative hypothesis $\left(H_{1}\right)$ is the opposite scenario of $H_{O}$. In the GFW group of a previous study [13], the mean change in MRS scores from baseline to day 30 was -7.27 ; the authors determined the mean change in MRS scores of the control group to be -1.45 under the assumption that it would correspond to approximately $20 \%$ of the value observed with the treatment group. With this previous study [13] as a reference, we assumed that the standard deviation of change in MRS scores from baseline to week 4 will be 6.73 . With a $5 \%$ significance level and $80 \%$ power, the sample size was determined to be 21 participants per group:

$$
\begin{aligned}
\mathrm{n} & =\frac{2\left(z_{a / 2}+z_{\beta}\right)^{2} \sigma^{2}}{\delta^{2}} \\
& =\frac{2(1.96+0.84)^{2}(6.73)^{2}}{(-5.82)^{2}} \quad 20.97 \approx 21
\end{aligned}
$$

Considering a $15 \%$ dropout rate, we determined that 25 participants will be ideally required per group.

\section{Statistical analysis}

The analysis set will comprise a full analysis set (FAS) and a per-protocol set (PPS). The FAS will include participants who satisfy the intention-to-treat principle as completely as possible. The PPS will include participants who are more compliant with the study protocol 
[31]. The FAS will be used for the main analysis. Analysis of covariance will be performed to evaluate the values of outcomes at weeks 5 and 9 as dependent variables, the baseline values as covariates, and the group as a constant. Participants will be divided into pre- and post-menopausal groups, which will be evaluated by exploratory subgroup analysis according to MRS scores in order to determine whether the intervention is equally effective on both subgroups. Intra-group comparisons will be performed by the paired $t$ test or Wilcoxon signed-rank test. The analyses will be performed considering an $\alpha$-value of $5 \%$ and a power of $80 \%$. Missing data will be imputed by multiple imputation. For socio-demographic characteristics, continuous variables will be presented as mean value and standard deviation, while categorical variables will be presented as frequency and percentage. Statistical analysis will be performed using the SAS program (version 9.4; SAS Institute Inc., Cary, NC, USA).

\section{Data and safety monitoring}

The trial data will be saved in an electronic data capture system (Medidata Rave; Medidata Solutions Inc., New York, NY, USA). Data quality will be ensured by regular monitoring. The monitoring agent will confirm whether the data are consistent with the source documents and whether the trial is conducted according to the approved protocol. All AEs observed during the study period will be recorded and reported.

\section{Discussion}

The purpose of this study is to explore the efficacy of GFW in the treatment of climacteric syndrome in women with blood stasis pattern. The study will also serve to monitor the safety of GFW.

Traditional Chinese medicine (TCM) has been adopted in a modified form in East Asian countries such as Japan and Korea [32, 33]. The system of pattern identification-the process of overall analysis of clinical data to determine the location, cause, and nature of disease in a patient and diagnosing a pattern/syndrome [34] - practiced in the three countries varies to a certain extent. In TCM, a practitioner recognizes the "Zheng" (clinical diagnosis based on analysis of medical history, symptoms, and signs) and confirms the treatment principle. In traditional Japanese medicine (Kampo), a practitioner chooses the most appropriate formula from among approximately 150 ready-to-use formulas, according to the "Sho" (pattern of symptoms) of the patient [32, 35]. Kampo can be characterized as a simplified and pragmatic version of Chinese herbal medicine [36]. In traditional Korean medicine (TKM), disease and pattern identification are made simultaneously as described in Dongui Bogam [37], which was compiled in the 17th century. TKM has been developed to include various modified methods involving the application of existing principles, methods, and formulas [38]. The diagnostic system of TKM includes the determination of diagnostic criteria based on indications for prescription of herbal medicines for a certain disease or its etiology [39-42]. Standardization of diverse pattern identification systems has been inadequate until now, which has made it challenging to apply pattern identification to clinical research on traditional medicine [43-46]. For the findings of clinical studies to be of direct aid in medical decision-making in traditional clinical practice, pattern identification systems used in routine practice need to be accurately reflected in the study design $[44,45]$. The present trial was planned in consideration of the target symptoms for herbal formulas, with Western medical methods for participant selection and outcome measurement. This approach is in agreement with a previous proposal for complementation of clinical research methods in traditional medicine [39].

Although the pattern identification and outcome measurement methods of this study are not ideal, they could serve as a reference for planning clinical trials that reflect the characteristics of traditional medicine. The results of this study will provide basic data for the designing of a large-scale trial evaluating the efficacy of GFW for the treatment of climacteric syndrome in women.

Trial status

Recruitment began in mid-October 2016.

\section{Additional file}

Additional file 1: SPIRIT 2013 checklist. (DOCX 51 kb)

Abbreviations

AE: Adverse event; ALP: Alkaline phosphatase; ALT: Alanine aminotransferase; ANCOVA: Analysis of covariance; AST: Aspartate aminotransferase; BUN: Blood urea nitrogen; Cr: Creatinine; E2: Estradiol; EDC: Electronic data capture; FAS: Full analysis set; FSH: Follicle-stimulating hormone; GFW: Guizhifulingwan; GMP: Good manufacturing practice; GTP: Glutamyl transpeptidase; Hb: Hemoglobin; Hct: Hematocrit; HRT: Hormone replacement therapy; Hs-CRP: High sensitivity C-reactive protein; ITT: Intention-to-treat; MRS: Menopause rating scale; PPS: Per-protocol set; RBC: Red blood cell; RCT: Randomized controlled trial; SAS: Strategic Applications Software; SPIRIT: Standard Protocol Items: Recommendations for Interventional Trials; TCM: Traditional Chinese medicine; TKM: Traditional Korean medicine; WBC: White blood cell WHOQOL: World Health Organization quality of life

\section{Acknowledgements}

Not applicable.

\section{Funding}

This study is supported by the Korea Institute of Oriental Medicine (K17121). The funder has no role in the design of the study, the collection, analysis, and interpretation of data, the writing of the manuscript, or the decision to submit the manuscript for publication.

Availability of data and materials Not applicable. 


\section{Authors' contributions}

JEK conceived and planned the study protocol and drafted the manuscript. $J H C$, JHK, MKK, and HYL participated in the design of the outcome measurements and helped to draft the manuscript. OJK planned the statistical design of the study and wrote part of the statistical methods. ARK, HJP, and SYJ provided technical advice and contributed to critical revision of the manuscript. $J H L$ participated in the overall design of the study, provided critical revision of the manuscript, and had the final responsibility for the decision to submit for publication. All authors read and approved the final manuscript.

\section{Competing interests}

The authors declare that they have no competing interests.

\section{Consent for publication}

Not applicable.

\section{Ethics approval and consent to participate}

This study has been approved by the institutional review boards of the Daejeon Oriental Hospital of Daejeon University (djomc-138-1). Written informed consent will be obtained from all participants by the investigator.

\section{Publisher's Note}

Springer Nature remains neutral with regard to jurisdictional claims in published maps and institutional affiliations.

\section{Author details}

${ }^{1}$ Clinical Research Division, Korea Institute of Oriental Medicine, 1672 Yuseongdae-ro, Yuseong-gu, Daejeon 34054, Republic of Korea. ${ }^{2}$ Department of Internal Korean Medicine, Daejeon Oriental Hospital of Daejeon University, 176-9, Daeheung-ro, Jung-gu, Daejeon 34929, Republic of Korea. ${ }^{3}$ University of Science \& Technology (UST), Korean Medicine Life Science, Daejeon 34054 , Republic of Korea.

\section{Received: 7 November 2016 Accepted: 3 March 2017}

Published online: 21 March 2017

\section{References}

1. Poomalar GK, Arounassalame B. The quality of life during and after menopause among rural women. J Clin Diagn Res. 2013;7:135-9.

2. North American Menopause Society. The 2012 hormone therapy position statement of the North American Menopause Society. Menopause. 2012;19:257-71.

3. Boardman HM, Hartley L, Eisinga A, Main C, Roqué i Figuls M, Bonfill Cosp X, et al. Hormone therapy for preventing cardiovascular disease in post-menopausal women. Cochrane Database Syst Rev. 2015:CD002229; doi: 10.1002/14651858. CD002229.pub4.

4. Collaborative Group on Epidemiological Studies Of Ovarian Cancer, Beral V, Gaitskell K, Hermon C, Moser K, Reeves G, et al. Menopausal hormone use and ovarian cancer risk: individual participant meta-analysis of 52 epidemiological studies. Lancet. 2015;385:1835-42.

5. Posadzki P, Lee MS, Moon TW, Choi TY, Park TY, Ernst E. Prevalence of complementary and alternative medicine (CAM) use by menopausal women: a systematic review of surveys. Maturitas. 2013;75:34-43.

6. Borrelli F, Ernst E. Alternative and complementary therapies for the menopause. Maturitas. 2010;66:333-43.

7. Liu WJ. What has been overlooked on study of Chinese materia medica in the West? Chin J Integr Med. 2015;21:483-92.

8. Cho KH, Kim YS, Jung WS, Kim TH. Effect of Gui-zhi-fu-ling-wan on hot flashes in young patients: a retrospective case series. J Acupunct Meridian Stud. 2011;4:129-33.

9. Park JS, Park S, Cheon CH, Jo SC, Cho HB, Lim EM, et al. Effects and safety of gyejibongnyeong-hwan on dysmenorrhea caused by blood stagnation: a randomized controlled trial. Evid Based Complement Alternat Med. 2013:2013:424730

10. Yoshino T, Katayama K, Horiba Y, Munakata K, Yamaguchi R, Imoto S, et al. The difference between the two representative Kampo formulas for treating dysmenorrhea: an observational study. Evid Based Complement Alternat Med. 2016;2016:3159617.

11. Terauchi M, Hiramitsu S, Akiyoshi M, Owa $Y$, Kato K, Obayashi $S$, et al. Effects of three Kampo formulae: Tokishakuyakusan (TJ-23), Kamishoyosan (TJ-24), and Keishibukuryogan (TJ-25) on Japanese peri- and postmenopausal women with sleep disturbances. Arch Gynecol Obstet. 2011;284:913-21.
12. Terauchi M, Akiyoshi M, Owa Y, Kato K, Obayashi S, Kubota T. Effects of the Kampo medication keishibukuryogan on blood pressure in perimenopausal and postmenopausal women. Int J Gynaecol Obstet. 2011;114:149-52.

13. Park JM, Yang JM, Kim DI. A clinical trial to verify the quality of life improvement efficacy of Dangguijakyak-san and Gyejibongnyeong-hwan granulation in postmenopausal women. J Orient Obstet Gynecol. 2007;20: 213-28 [Article in Korean].

14. Ushiroyama T, Ikeda A, Sakuma K, Ueki M. Comparing the effects of estrogen and an herbal medicine on peripheral blood flow in postmenopausal women with hot flashes: hormone replacement therapy and gui-zhi-fu-ling-wan, a Kampo medicine. Am J Chin Med. 2005;33:259-67.

15. Namiki T, Sato $H$, Matsumoto $Y$, Kakikura $H$, Ueno $K$, Chino $A$, et al. Identification of a predictive biomarker for the beneficial effect of keishibukuryogan, a Kampo (Japanese traditional) medicine, on patients with climacteric syndrome. Evid Based Complement Alternat Med. 2014:2014:962109.

16. Plotnikoff GA, Watanabe K, Torkelson C, La Valleur J, Radosevich DM. The TU-025 keishibukuryogan clinical trial for hot flash management in postmenopausal women: results and lessons for future research. Menopause. 2011;18:886-92.

17. Hauser GA, Huber IC, Keller PJ, Lauritzen C, Schneider HPG. Evaluation der klinischen Beschwerden (Menopause Rating Scale). Zentralbl Gynakol. 1994;116:16-23 [Article in German]

18. Potthoff $P$, Heinemann LAJ, Schneider HPG, Rosemeier HP, Hauser GA. Menopause-Rating Skala (MRS): Methodische Standardisierung in der deutschen Bevölkerung. Zentralbl Gynakol. 2000;122:280-6.

19. International Menopause Society. Menopause terminology. http://www. imsociety.org/menopause_terminology.php. Accessed 20 June 2016.

20. Witt CM, Linde K. Clinical research in complementary and integrative medicine: a practical training book. 1st ed. Seoul: Elsevier Korea; 2013 [Book in Korean].

21. Bang HJ, Ni LY, Davis CE. Assessment of blinding in clinical trials. Control Clin Trials. 2004;25:143-56

22. The WHOOOL Group. Development of the World Health Organization WHOQOL-BREF quality of life assessment. Psychol Med. 1998;28:551-8

23. Min SK, Lee Cl, Kim KI, Suh SY, Kim DK. Development of Korean version of WHO quality of life scale abbreviated version (WHOQOL-BREF). J Korean Neuropsychiatr Assoc. 2000;39:571-9 [Article in Korean].

24. Min SK, Kim Kl, Park IH. Korean version of WHOQOL. 1st ed. Seoul: Hana Medical Press; 2002 [Book in Korean].

25. Shigetaro K. Abdominal examination and prescription. 2nd ed. Wonju: Uibang Press; 2014 [Book in Korean].

26. Roh YB. Abdominal examination and authentic prescription. 1st ed. Goyang: Daesung Medical Press; 2006 [Book in Korean]

27. Shibata Y, Wu J. Kampo treatment for climacteric disorders. 1st ed. Brookline: Paradigm Publications; 1997.

28. Ahn JH, Lee SH, Park YJ, Park YB. Developing questionnaire of BiJeung (痺證) which is similar to arthralgia and paresthesia syndrome. J Korean Med. 2013:34:96-105 [Article in Korean].

29. Yang DH, Park YJ, Park YB, Lee SC. Development of questionnaires for blood stasis pattern. J Korean Orient Med Diagn. 2006;10:141-52 [Article in Korean]

30. Seo SM, Baek SH, Jeon HK, Kang SM, Kim DS, Kim WS, et al. Correlations between the level of high-sensitivity C-reactive protein and cardiovascular risk factors in Korean adults with cardiovascular disease or diabetes mellitus: the CALLISTO study. J Atheroscler Thromb. 2013;20:616-22.

31. Lewis JA. Statistical principles for clinical trials (ICH E9): an introductory note on an international guideline. Stat Med. 1999;18:1903-42.

32. Yu F, Takahashi T, Moriya J, Kawaura K, Yamakawa J, Kusaka K, et al. Traditional Chinese medicine and Kampo: a review from the distant past for the future. J Int Med Res. 2006:34:231-9.

33. Park J, Park HJ, Lee HJ, Ernst E. What's in a name? A systematic review of the nomenclature of Chinese medical formulae. Am J Chin Med. 2002;30:419-27.

34. WHO Regional Office for the Western Pacific. WHO International Standard Terminologies on Traditional Medicine in the Western Pacific Region. Geneva: World Health Organization; 2007.

35. Scheid V, Ward T, Cha WS, Watanabe K, Liao X. The treatment of menopausal symptoms by traditional East Asian medicines: review and perspectives. Maturitas. 2010;66:111-30.

36. Terasawa K. Evidence-based reconstruction of Kampo medicine: Part I - Is Kampo CAM? Evid Based Complement Alternat Med. 2004;1:11-6. 
37. Heo J. Translated Dongui Bogam. 1st ed. Seoul: Bubin Publishers Co.; 1999 [Book in Korean].

38. Chi GY. A research on it's teaching way for efficient lecture about differential diagnostic theory and method. Korean J Orient Physiol Pathol. 1998;12:8-13 [Article in Korean].

39. Jung KY, Lee MH, Choi YK, Lee CY, Park JH, Jeon CY. Study on the proposals for clinical research in Korean medicine worldwide - future clinical research strategy II. J Physiol Pathol Korean Med. 2015;29:115-26 [Article in Korean].

40. Lee SD, Kim EJ, Jung CY, Shin KM, Jang MK, Yoon EH, et al. Selection of adequate indicators for the development of a questionnaire to evaluate the effects of Ojeock-san. J Korean Orient Med. 2010;31:101-14 [Article in Korean]

41. Koo BS, Lee SJ, Han CH, Kim HJ, Park SH. The basic study for building the depression prescription guideline of Gamiguibi-Tang — the evaluation of reliability and validity of the depression pattern-identification questionnaire. J Orient Neuropsychiatry. 2009;20:1-13 [Article in Korean]

42. Lim JS, Oh HS, Lee SC, Park YB. Developing questionnaire for pathogenesis analysis of Pyungweesan symptom. J Korean Orient Med Diagn. 2007;1 1:72-81 [Article in Korean].

43. Jang ES, Kim YY, Lee EJ, Yoo HR, Jung IC. Review on the development state and utilization of pattern identification questionnaire in Korean medicine by $\mathrm{U}$ code of Korean Classification of Disease. J Physiol Pathol Korean Med. 2016;30:124-30 [Article in Korean].

44. Jung KY, Go HY, Sun SH, Jeong JJ, Park JS, Song YK, et al. Study on the current situation and issues for clinical research in Korean medicine worldwide - future clinical research strategy I. Korean. J Orient Physiol Pathol. 2014;28:137-45 [Article in Korean]

45. Watanabe K, Matsuura K, Gao P, Hottenbacher L, Tokunaga H, Nishimura K, et al. Traditional Japanese Kampo medicine: clinical research between modernity and traditional medicine- the state of research and methodological suggestions for the future. Evid Based Complement Alternat Med. 2011;2011:513842.

46. Shea JL. Applying evidence-based medicine to traditional Chinese medicine: debate and strategy. J Altern Complement Med. 2006;12:255-63.

\section{Submit your next manuscript to BioMed Central and we will help you at every step:}

- We accept pre-submission inquiries

- Our selector tool helps you to find the most relevant journal

- We provide round the clock customer support

- Convenient online submission

- Thorough peer review

- Inclusion in PubMed and all major indexing services

- Maximum visibility for your research

Submit your manuscript at www.biomedcentral.com/submit

) Biomed Central 